\title{
Prosthetic Rehabilitation of anopthalmic socket: Two case reports
}

\author{
Prema $^{1}$, Sounder Raj K ${ }^{2}$, Anu Raveendran ${ }^{3 *}$, Preeti $\mathbf{M}^{4}$ \\ ${ }^{1}$ Associate Professor, ${ }^{2}$ Professor and Head, ${ }^{3,4}$ Post Graduate, Dept. of Prosthodontics, Government Dental College, \\ Bangalore, Karnataka, India
}

*Corresponding Author: Anu Raveendran

Email: anu_12_91@yahoo.co.in

\begin{abstract}
An ocular prosthesis is a simulation of human anatomic eyeball to create an illusion of a normal healthy eye and surrounding structures. So the perfect replication of colour, contour and size of the contralateral eye is essential to provide realism and symmetry for the patient. In addition, precise recording of the anopthalmic socket is also essential for the proper retention and form of prosthesis. This article aims to describe the prosthetic rehabilitation of two patients after enucleation surgery with custom made ocular prosthesis.
\end{abstract}

Keywords: Anopthalmic socket, Enucleation, Eye prosthesis, Ocular defect, Ocular prosthesis

\section{Introduction}

The human body's window to the outside world, the eye is a complex optical system whose importance is not restricted to vision alone. Loss of damage to an eye can have a crippling effect on the physical appearance as well as the day-to-day activities of the individual concerned. The psychological effect of this loss or damage to a vital organ can far outweigh the physical effect.

A congenital defect, pathology or accidental trauma may necessitate surgical intervention which may lead to the removal of the eyeball. ${ }^{1}$ The treatment involved can vary from a minimal surgical intervention such as evisceration or enucleation to extensive radical treatment such as exenteration. ${ }^{2}$ An ocular prosthesis acts as a synthetic substitute for the missing eye. ${ }^{3}$

\section{Clinical Report}

\section{Case 1}

A 24- year old female patient with a missing right eye was referred to the Department of Prosthodontics GDCRI Bangalore. A history of traumatic perforating injury resulting in enucleation of the right eye was recorded. The procedure was performed at Minto Eye Hospital, Bangalore. The patient was planned for a custom ocular prosthesis.

\section{Impression procedure}

An impression of the anopthalmic socket was made following Allen and Webster technique. ${ }^{4}$ A stock ocular impression tray was selected and was fit into the confines of the socket and checked for its extension. Overextensions were trimmed and margins were finished using a finishing bur. During the procedure, the patient was made to sit upright with the head supported and was asked to look straight.

Loose consistency alginate (Algitex; Dental Products of India Mumbai) was injected into the impression tray using a syringe through the inlet of the stock ocular impression tray. In order to increase the working time of the alginate, cold water was used. A little amount of alginate overflowing through the inner canthus of the eye indicates adequate material filling the socket. After the impression was set, the lower eyelid was held downwards and the impression tray was removed by sliding it out from the upper eyelid.

The impression was checked for an accurate recording of the posterior wall, the position of palpebrae in relation to the posterior wall, and the greatest extent of superior and inferior fornices of the palpebrae denoting precise impression.

\section{Scleral Wax Pattern Fabrication}

The ophthalmic impression was immersed in a medicine cup filled with irreversible hydrocolloid. A different colour alginate impression material was used 
for pouring the impression to differentiate the impression and the mould while retrieval. Once set, an incision was made on the sides of the mould with a sharp scalpel. The mould was spread apart and the impression tray was removed. The scleral wax pattern was fabricated by pouring a combination of inlay wax and baseplate wax into the mould to have sufficient rigidity for carving and handling. On getting set, the wax pattern was gently retrieved, carved and smoothened using a gauze piece and a carver.

\section{Scleral wax pattern trial}

The cleaned and polished wax pattern was tried in the patient's eye. The patient was then asked to perform various eye movements. The fit, bulk, aesthetics, and the ability to open and close the eyelids were evaluated. There were no deficiencies in the extension of the scleral wax pattern. The support and contour of the wax pattern were also checked by bimanual palpation with eyes closed as well as visually by matching it with the contralateral eye.

\section{Iris positioning}

The iris positioning was done using an aluminium iris button by matching it to the contralateral eye. The patient was instructed to gaze straight at a fixed point at a distance. The patient was then asked to perform various eye movements and the movement of the iris eye button was matched to the contralateral eye. The iris positioning was again verified by measuring the distance from inner canthus to iris. An iris button closely resembling the contralateral eye in terms of colour, size, and shape was selected and placed in the marked position.

\section{Investing, dewaxing, and packing}

To secure its position and prevent its displacement during dewaxing, a groove was marked around the iris button in the wax pattern. The wax pattern was then invested in dental plaster by two-pour technique and later subjected to dewaxing. After dewaxing, the iris button was secured in position and white coloured acrylic was packed into the mould and later acrylised.

The processed ocular prosthesis was retrieved, finished, and reduced at about $1 \mathrm{~mm}$ on the external surface. The inner surface of the prosthesis was polished and tried on the patient. At this stage, the extension, fit and support were checked.

The characterization of the scleral shell was done using suitable stains in the presence of the patient and was matched with the contralateral eye. Red nylon fibres resembling blood vessels were added to give it a natural look. After colour matching, the acrylic shell was packed with clear acrylic resin and was subsequently cured again. The prosthesis was retrieved, finished and margins were rounded off. It was polished using buff and pumice to give it a natural look.

The final ocular prosthesis was tried in the anophthalmic socket. Home care instructions regarding the insertion and removal of the prosthesis, proper care and hygiene were imparted to the patient. Follow-up evaluation was performed once in three months, and the prosthesis was found to be functioning well.

\section{Case 2}

A 16-year-old patient complaining of a missing left eye was referred to the aforementioned department. The patient's history revealed a traumatic injury at the age of eight followed by lens atrophy and cystic transformation. An enucleation of the left eye was done. The fabrication procedure followed here was similar to that in Case 1. After the acrylisation of the ocular prosthesis, colour matching and extrinsic staining were completed. The colour matching was done in daylight. Veining was carried out with red nylon fibres and the prosthesis was tried in the patient's socket. Post insertion instructions were given.

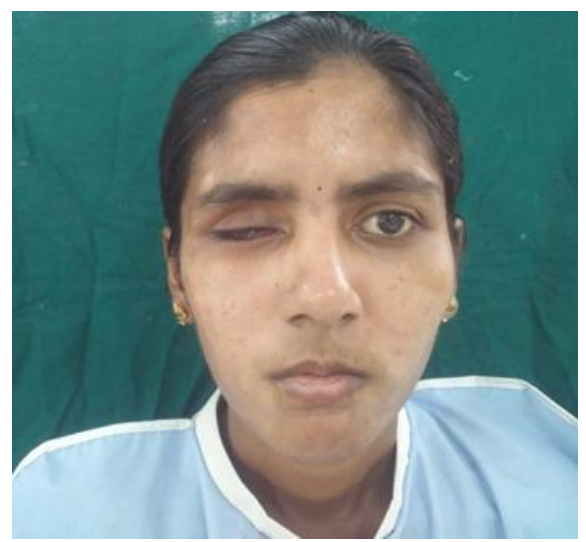

Fig. 1: Prior to treatment 


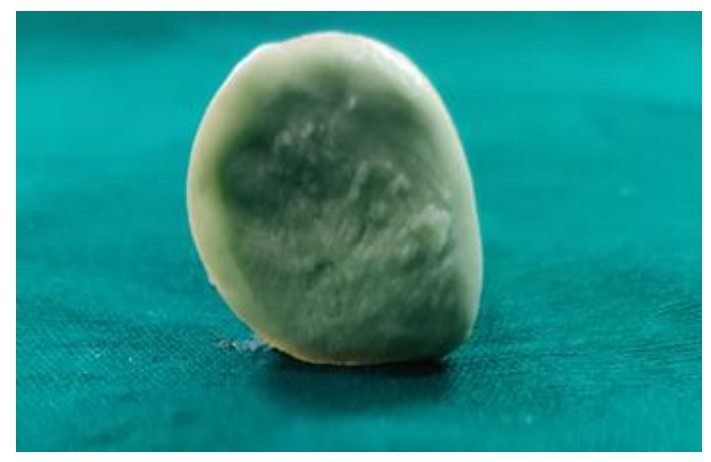

Fig. 2: Ocular impression

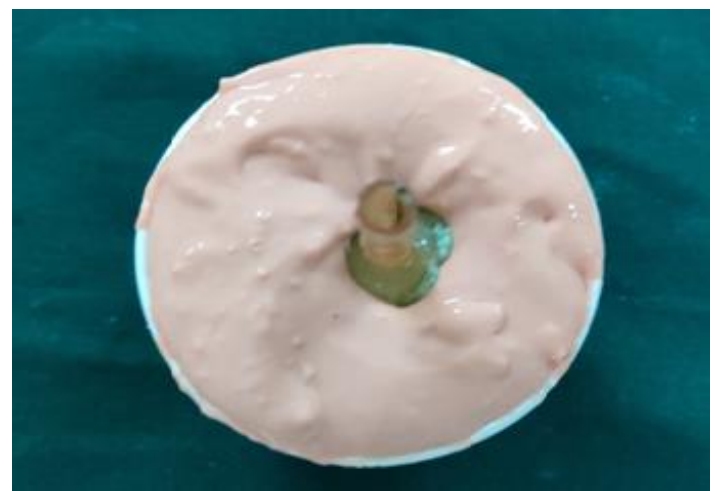

Fig. 3: Impression poured in a different colour alginate

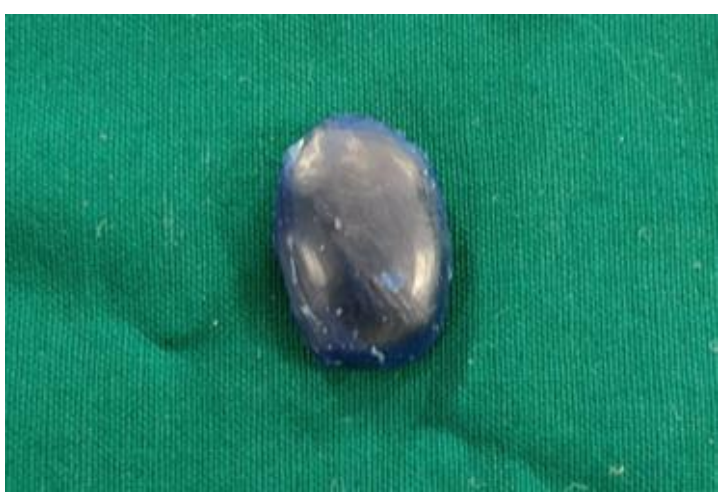

Fig. 4: Scleral wax pattern

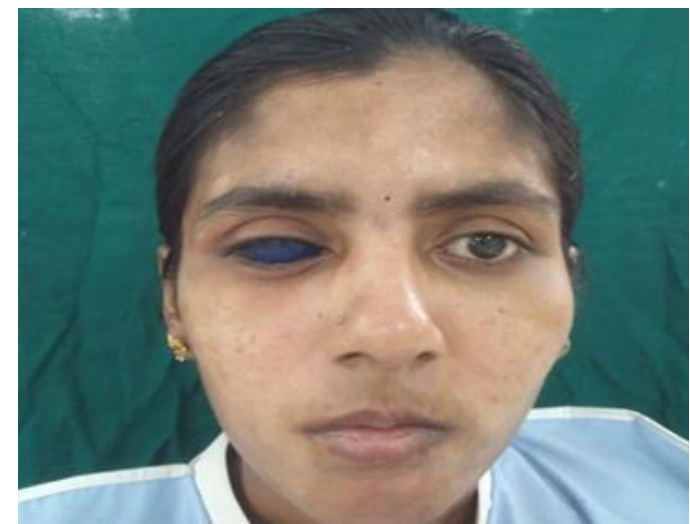

Fig. 5: Scleral wax pattern tried in the patient's eye

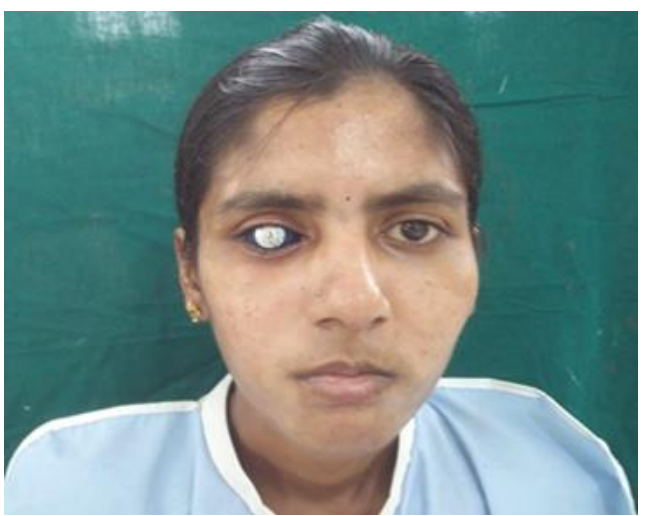

Fig. 6: Iris positioning done using aluminum button

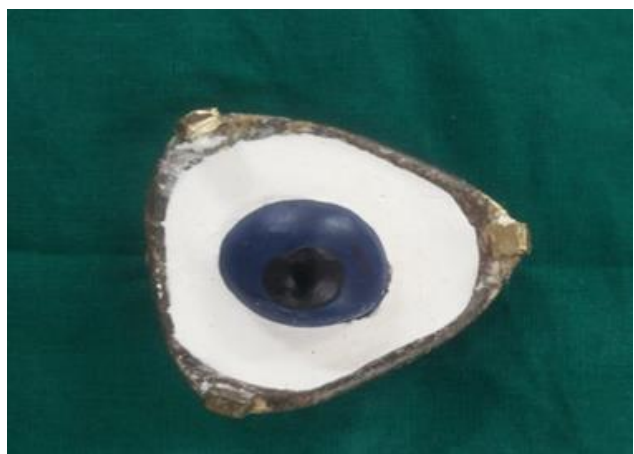

Fig. 7: Invested scleral wax pattern

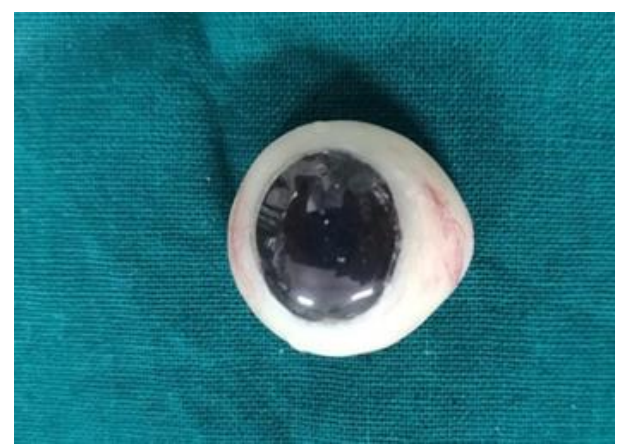

Fig 8: Final ocular prosthesis

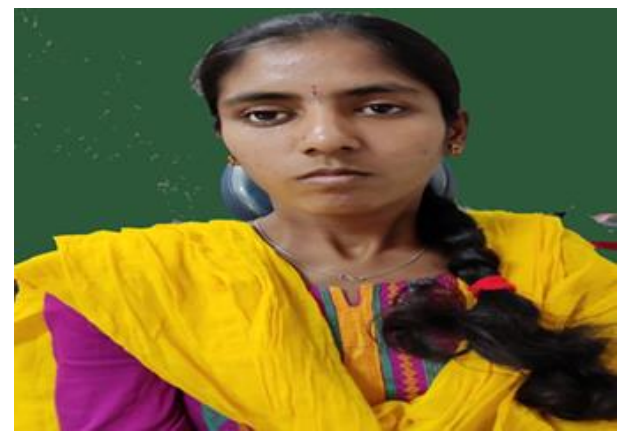

Fig. 9: Post treatment 

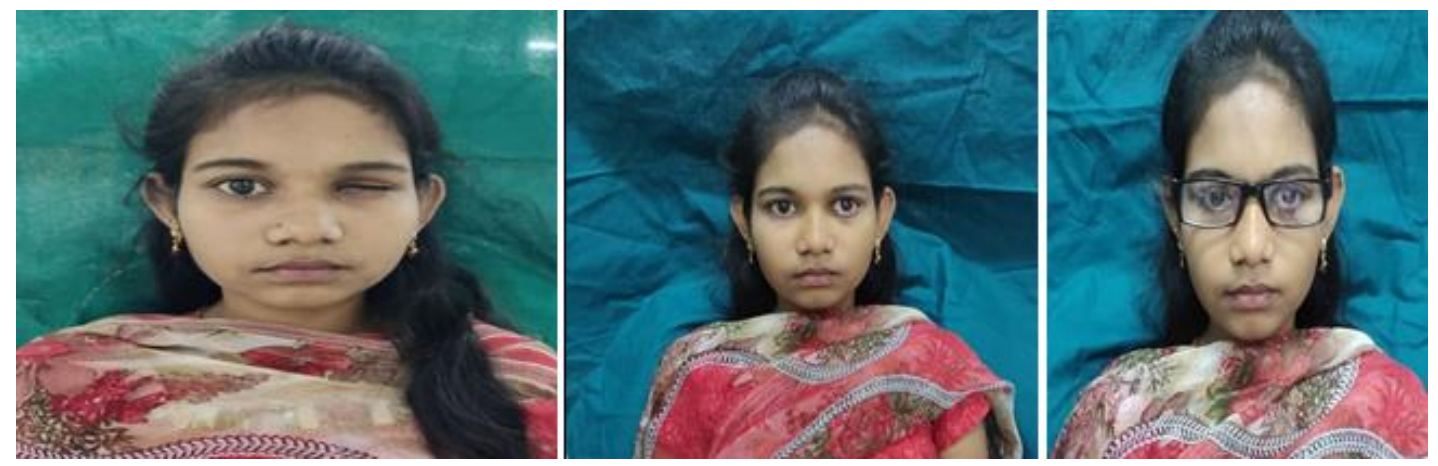

Fig. 10,11,12: Prior and post treatment in Case 2

\section{Discussion}

This case report describes the prosthetic rehabilitation of two enucleated sockets with an ocular prosthesis. Surgical procedures for removal of an eye are classified by Peyman, Saunders, and Goldberg (1987) into three categories as Evisceration (where the contents of globe are removed leaving the sclera intact), Enucleation (most common where the entire eyeball is removed after severing the muscle and optic nerve) and Exenteration (where the entire contents of the orbit including eyelid and surrounding tissues are removed). ${ }^{4}$

Cosmetic rehabilitation of such afflicted patients by way of an ocular prosthesis helps them gain professional and social acceptance apart from alleviating other problems. A custom ocular prosthesis is preferred as it has the advantage of close adaptation to the tissue bed, provides maximum comfort and restores full physiologic function to accessory organs of the eye. ${ }^{6}$

\section{Conclusion}

In this case report, a simple technique for the fabrication of ocular prosthesis was described. This technique is cost-effective, affordable and one which can be carried out in a small clinical setup. This method provides good results in terms of retention, aesthetics, and function.

\section{Source of Funding}

None

\section{Conflict of Interest}

None

\section{References}

1. Kathuria N, Prasad R, Gupta N, Gulati M, Bhide SV. A modified technique and simplified laboratory procedure for Ocular Prosthesis Fabrication. J Prosthod Res 2012;56(2):147-50.

2. Shetty PP, Chowdhary R, Yadav RK, Gangaiah M. An iris positioning device and centering approach: A technique. $J$ Prosthetic Dent 2018;119(1):175-7.

3. Abbas SK, Fatihallah AA, Ali MM. Matching the Iris Color of Ocular Prosthesis Using an Eye Contact Lens: New Technique. Iraqi Dent J 2017;39(1):23-5.

4. Allen L, Webster HE. Modified impression method of artificial eye fitting. Am J Opthalmol 1969;67:189-218

5. Agrawal KK, Mall P, Alvi H A, Rao J, Singh K. Fabrication of custom made eye prosthesis for anophthalmic paediatric patients: 2 case reports. $J$ Interdiscip Dent 2012;2:128-31

6. Benson P. The fitting and fabrication of a custom resin artificial eye. J Prosthet Dent 1977;38:532-8.

How to cite this article: Prema, Raj SK, Raveendran A, Preeti M. Prosthetic Rehabilitation of anopthalmic socket: Two case reports. Ann Prosthodont Restor Dent 2019;5(4):121-4. 